\title{
Highlights from the NA61/SHINE experiment at the CERN SPS
}

\author{
Katarzyna Grebieszkow* for the NA61/SHINE Collaboration \\ Warsaw University of Technology \\ E-mail: kperl@if.pw.edu.pl
}

The NA61/SHINE experiment at the CERN SPS aims to study hadron production in hadron+hadron, hadron+nucleus and nucleus+nucleus interactions. Within the NA61 ion program we plan to study the properties of the onset of deconfinement and search for the signatures of the critical point. This will be achieved by performing a two-dimensional scan of the $\left(T-\mu_{B}\right)$ phase diagram by varying the energy $(13 A-158 A \mathrm{GeV})$ and the system size $(p+p, B e+B e$, $\mathrm{Ar}+\mathrm{Ca}, \mathrm{Xe}+\mathrm{La}$ ) of the collision.

The main motivation of the NA61 neutrino program is to obtain precision data on hadron production (spectra) in $p+C$ interactions. They serve as reference measurements for the T2K experiment for computing neutrino fluxes from the T2K beam targets. Finally, hadron spectra in $p+C, p+p$, $\pi+C$, and $K+C$ will be used as reference measurements in cosmic-ray physics (Pierre-Auger and KASCADE experiments) for improving air shower simulations.

This paper reviews the status, results, and plans of the NA61/SHINE experiment. In particular the detector upgrades and data taking schedule are discussed and the progress of the data analysis is illustrated.

8th International Workshop on Critical Point and Onset of Deconfinement

March 11 - 15, 2013

Napa, California, USA

\footnotetext{
*Speaker.
} 


\section{NA61/SHINE detector and physics program}

NA61/SHINE ${ }^{1}$ (see [1] for a set-up) is a fixed target experiment in the north area of the CERN SPS. The main devices, namely four large volume Time Projection Chambers (TPC) (two of them, Vertex TPCs (VTPC), inside superconducting magnets and two others, Main TPCs (MTPC), downstream of the magnets and symmetric to the beam line) and two Time-of-Flight (ToF) walls were inherited from NA49 [2]. Several

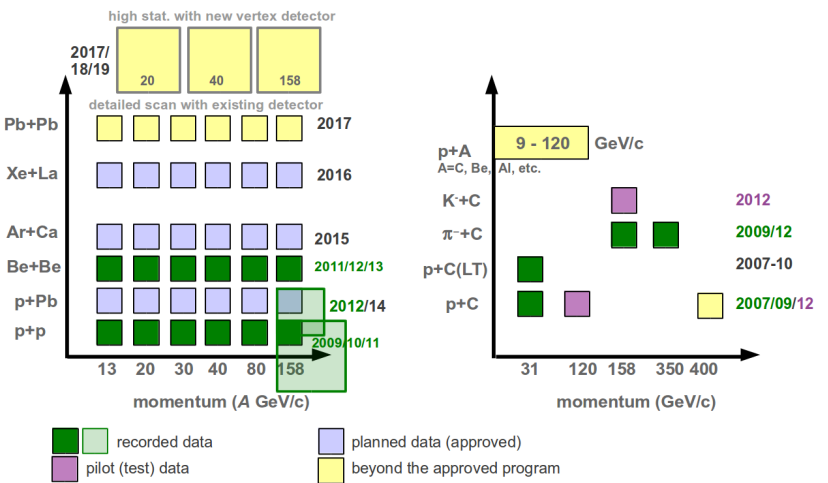

Figure 1: Status of the NA61/SHINE data taking within the heavy ion program (left) and within the neutrino and cosmic-ray physics programs (right). upgrades were completed by the NA61 Collaboration. An additional forward ToF wall was constructed to extend ToF acceptance for particles with $p<3 \mathrm{GeV} / \mathrm{c}$. The NA49 Forward Calorimeter was replaced by the Projectile Spectator Detector (PSD) with transverse segmentation and with a five times better energy resolution corresponding to one nucleon(!) in the studied energy range. Such precise measurement of the energy of projectile spectators is needed for a tight centrality selection, as required for the analysis of fluctuations. Channeling of a high intensity heavy ion beam through the gas volume of the VTPC has limitations when compared to a proton beam. Delta electrons produced in the gas volume inside the VTPCs from heavy ion beam-gas interactions significantly increase the background in the TPCs (particularly for heavy nuclei) and distort measurements of event-by-event fluctuations. Therefore, helium filled beam pipes were installed inside both VTPCs (around the beam line) resulting in a reduction of the $\delta$-electron background by a factor of 10 .

In NA61 we record data with numerous types of beams, i.e. ions (secondary $\mathrm{Be}$ and primary $\mathrm{Ar}$ and $X e$ ) at beam momentum $13 A-158 A \mathrm{GeV} / \mathrm{c}$, and (secondary) hadrons ( $p$ at $13-158 \mathrm{GeV} / \mathrm{c}, \pi^{-}$ at 158 and $350 \mathrm{GeV} / \mathrm{c}, K^{-}$at $158 \mathrm{GeV} / \mathrm{c}$ ). The NA61/SHINE program studies hadron production in $p+p, p+A, h+A$, and $A+A$ at various energies. The NA61 physics program covers: heavy and light ion collisions (spectra, fluctuations and correlations are analyzed in order to search for the critical point and study the properties of the onset of deconfinement), study of high $p_{T}$ particle production (energy dependence of the nuclear modification factor), and precision measurements of hadron production for improving simulations of $v$ beam properties at J-PARC for the T2K experiment, and for improving air shower simulations for the Pierre-Auger and KASCADE cosmic-ray experiments. We also consider extensions beyond the approved NA61 program, including the measurements of $\mathrm{Pb}+\mathrm{Pb}$ collisions for the ion program (open charm and multi-strange particle production using a new vertex detector, and extension of high $p_{T}$ spectra), measurements for the Fermilab neutrino program, and measurements for the CERN (LBNO) neutrino program. The current status of data taking of NA61 is presented in Fig. 1.

\footnotetext{
${ }^{1}$ SHINE - SPS Heavy Ion and Neutrino Experiment
} 


\section{Results for neutrino and cosmic-ray physics experiments}

\subsection{Reference measurements by NA61/SHINE for neutrino physics}

NA61 provides high quality measurements of hadron production to precisely predict the neutrino flux at J-PARC. The T2K experiment uses $p+C \rightarrow \pi, K, \cdots \rightarrow v_{\mu}, \cdots$ reactions to produce the neutrino beam, and observes interactions of the $v \mathrm{~s}$ in near detectors $(280 \mathrm{~m})$ and a far detector (Super Kamiokande) $(295 \mathrm{~km})$. The T2K experiment is looking for $v$ oscillations $\left(v_{\mu} \rightarrow v_{e}\right)$, which are obtained from far-to-near flux ratios. The corrections and systematic error estimates depend on the precision with which $p+C \rightarrow \pi^{+-}, K^{+-}+X$ production is known. The NA61 experiment reduces these uncertainties by new measurements of the reactions $p+C$ at $31 \mathrm{GeV} / \mathrm{c}$ and $p+C(L T)$ at $31 \mathrm{GeV} / \mathrm{c}$, where $L T$ is a $90 \mathrm{~cm}$ long T2K replica target.

The T2K experiment published a measurement of the angle $\Theta_{13}$ in the neutrino mixing matrix [3]. The systematic error estimate was based on the NA61/SHINE results on pion production in $p+C$ interactions at $31 \mathrm{GeV} / \mathrm{c}$ [4]. The published pion spectra [4] were used not only to improve beam neutrino flux predictions but also to adjust particle production models (UrQMD [5], Fritiof [6]) used in neutrino and cosmic-ray experiments. The NA61 experiment also published positively charged kaon spectra in $p+C$ interactions at $31 \mathrm{GeV} / \mathrm{c}$ [7], which are used by the T2K experiment for precise prediction of the high energy tail and the $v_{e}$ content of their $v$ beam.

A large fraction (up to $40 \%$ ) of the neutrinos originates from particles produced by re-interactions of secondary particles in the target, which for $\mathrm{T} 2 \mathrm{~K}$ is $90 \mathrm{~cm}$ long. This contribution is difficult to calculate precisely, motivating a careful analysis of the data taken with the long target. The recently obtained NA61 pion spectra in $p+C(L T)$ collisions at $31 \mathrm{GeV} / \mathrm{c}$ (T2K replica target) result in values of neutrino flux very similar to those based on data from $p+C$ at $31 \mathrm{GeV} / \mathrm{c}$ (thin target) [8].

Preliminary NA61 results on $K_{S}^{0}$ and $\Lambda$ spectra in $p+C$ interactions at $31 \mathrm{GeV} / \mathrm{c}$ are shown in Fig. 2. $K_{S}^{0}$ production is measured to better constrain the $v_{e}$ and $\bar{v}_{e}$ flux at high momenta, and to reduce model dependence of feed-down corrections ( $\pi^{-}$from $\Lambda$ and $K_{S}^{0}$ decays). In production processes of $p+C$ interactions at $31 \mathrm{GeV} / \mathrm{c}$ we obtained the average multiplicity $\left\langle K_{S}^{0}\right\rangle=0.125 \pm$ 0.007 (stat) $\pm 0.020($ sys $)$, and the inclusive cross section $\sigma\left(K_{S}^{0}\right)=28.58 \pm 1.85($ stat $) \pm 4.62($ sys $)$ $[\mathrm{mb}]$.

\subsection{Reference measurements by NA61/SHINE for cosmic-ray physics}

The NA61 cosmic-ray physics program aims at a measurement of particle production spectra for the interpretation of air shower observables measured at e.g. the Pierre Auger Observatory, IceTop or the KASCADE-Grande array. These experiments study high-energy cosmic rays. The primary cosmic ray is reconstructed from the measured properties of the air shower. The main problems are large systematic uncertainties of models used for air shower simulations. The measured shower properties strongly depend on the $\pi^{+-}+$air nucleus $\rightarrow \pi^{+-}, K^{+-}, p, \cdots$ production at the SPS energies (energies of the last generation of air shower cascade). Special "cosmic runs" of $\pi^{-}+C$ at $158 \mathrm{GeV} / \mathrm{c}$ and $350 \mathrm{GeV} / \mathrm{c}$ were registered by NA61 (carbon is close to mass of air nuclei). For this program $p+p$ data at $13-158 \mathrm{GeV} / \mathrm{c}$ and $p+C$ collisions at $31 \mathrm{GeV} / \mathrm{c}$ will also be useful.

Charged hadron $\left(h^{+}\right.$and $h^{-}$) spectra in $\pi^{-}+C$ interactions at $158 \mathrm{GeV} / \mathrm{c}$ and $350 \mathrm{GeV} / \mathrm{c}$ beam momentum have recently been released (see examples for selected momentum bins in Fig. 3). They 

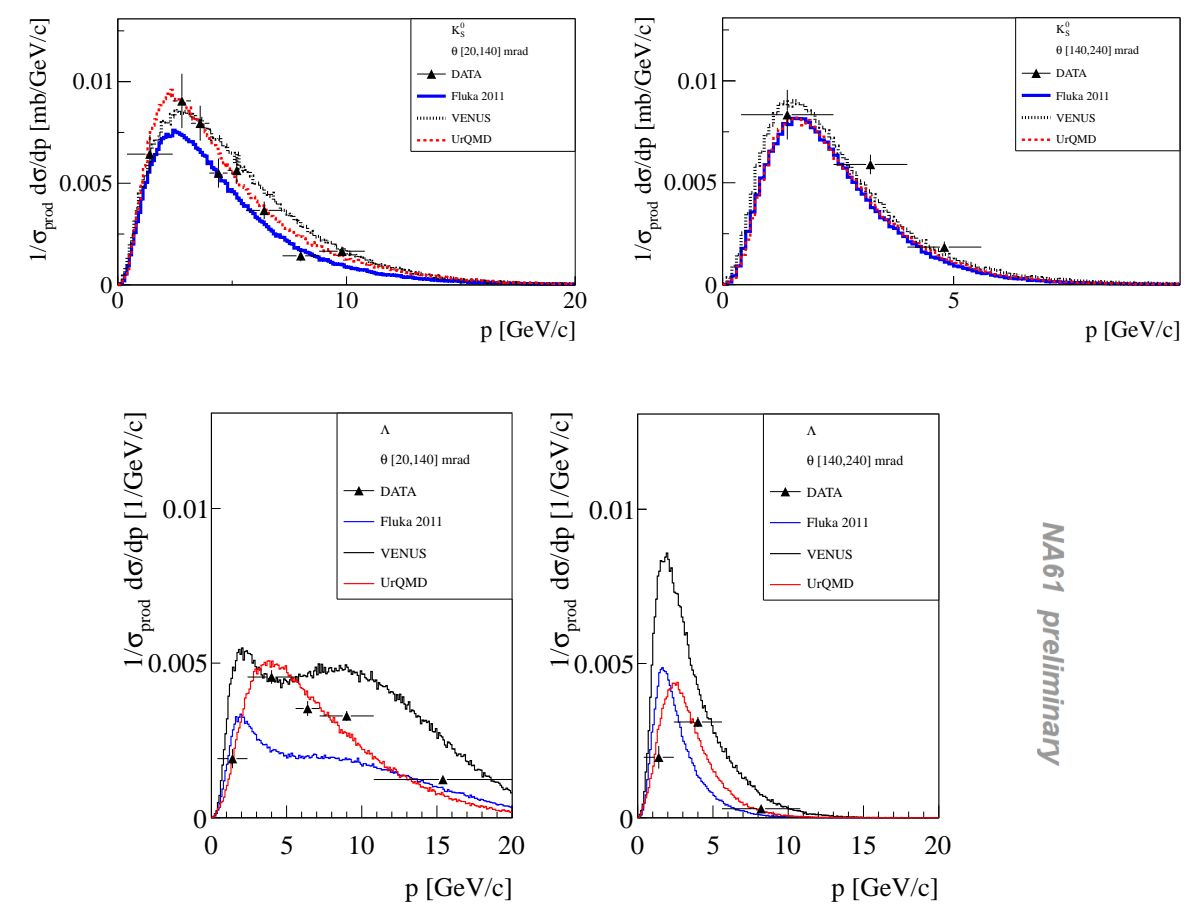

Figure 2: Preliminary $K_{S}^{0}$ and $\Lambda$ total momentum distributions for two different polar angle bins measured in $p+C$ collisions at $31 \mathrm{GeV/c}$. Only statistical errors are shown.
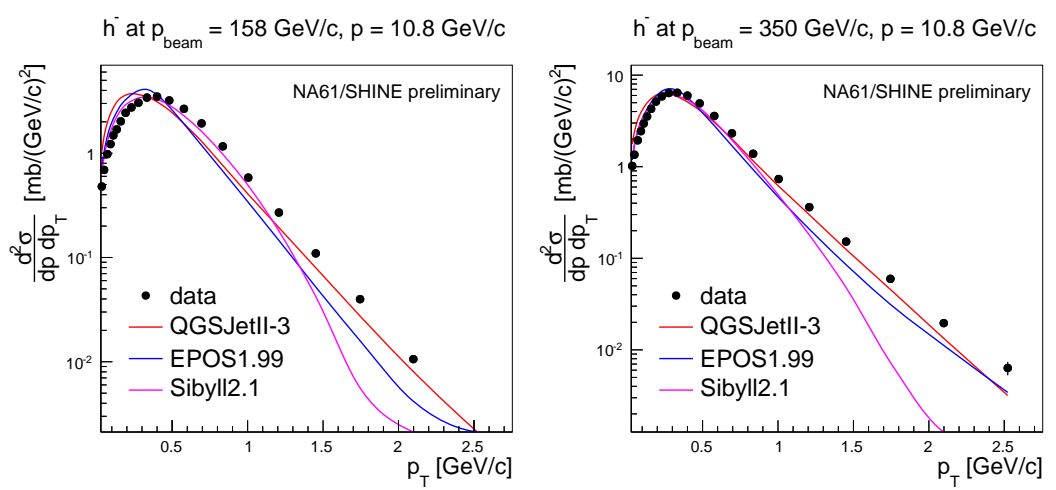

Figure 3: Examples of transverse momentum spectra of negatively charged hadrons produced in $\pi^{-}+C$ interactions at $158 \mathrm{GeV} / \mathrm{c}$ and $350 \mathrm{GeV} / \mathrm{c}$ beam momentum.

have been already compared to several models (EPOS, UrQMD, Fluka, QGSJet, etc.) and are important inputs for validation/tuning of these Monte Carlo generators.

\section{Results from the heavy ion program}

The energies of the CERN SPS accelerator cover a very important region of the phase diagram of strongly interacting matter. First, the NA49 experiment showed that the energy threshold for deconfinement (minimum energy to create a partonic system) is located at low SPS energies $\left(\sqrt{s_{N N}} \approx 7.6 \mathrm{GeV}\right.$ or $30 \mathrm{~A} \mathrm{GeV}$ ) [9]. Second, theoretical calculations suggest that the critical point $(\mathrm{CP})$ of strongly interacting matter is located at energies accessible at the CERN SPS. 
Examples of predictions are: $T^{C P}=162 \pm 2 \mathrm{MeV}, \mu_{B}^{C P}=360 \pm 40 \mathrm{MeV}[10]$ or $\left(T^{C P}, \mu_{B}^{C P}\right)=$ $\left(0.927(5) T_{c}, 2.60(8) T_{c}\right)=(\sim 157, \sim 441) \mathrm{MeV}[11]$, or $\left(T^{C P} / T_{c}, \mu_{B}^{C P} / T^{C P}\right)=(\sim 0.96, \sim 1.8)$ $\left(\mu_{B} \sim 290 \mathrm{MeV}\right)$ [12], where $T_{c}$ is the cross-over temperature at $\mu_{B}=0$.

NA61 will perform a comprehensive scan in the whole SPS beam momentum range (13A$158 \mathrm{~A} \mathrm{GeV/c)}$ with light and intermediate mass nuclei $(p, B e, A r, X e)$. This will allow to cover a wide region of the phase diagram (the expected chemical freeze-out points are shown in Fig. $4^{2}$ ). The properties of the onset of deconfinement will be studied by looking for the kink, horn, step, and dale structures $[14,15]$ in collisions of light nuclei (the structures observed for $P b+P b / A u+A u$ should vanish with decreasing system size). The NA61 data will also allow us to search for the critical point. An increase of CP-sensitive fluctuation measures (hill of fluctuations) is expected for systems freezing-out near the CP [16]. Therefore, non-monotonic dependence of fluctuation signals (multiplicity and average $p_{T}$ fluctuations, intermittency, etc.) on control parameters (energy and ion size) can help to locate the CP.

\subsection{Spectra and yields in $p+p$ collisions}

Spectra and yields in $p+p$ interactions were obtained from two different methods. The $h$-analysis method allows to obtain $\pi^{-}$spectra assuming that the majority of produced negatively charged particles are pions. Contributions of other particles (mostly $K^{-}$and feed-down) were subtracted using the average of VENUS and EPOS Monte-Carlo model predictions. This approach allows to obtain $\pi^{-}$ spectra in full measured phase-space in a

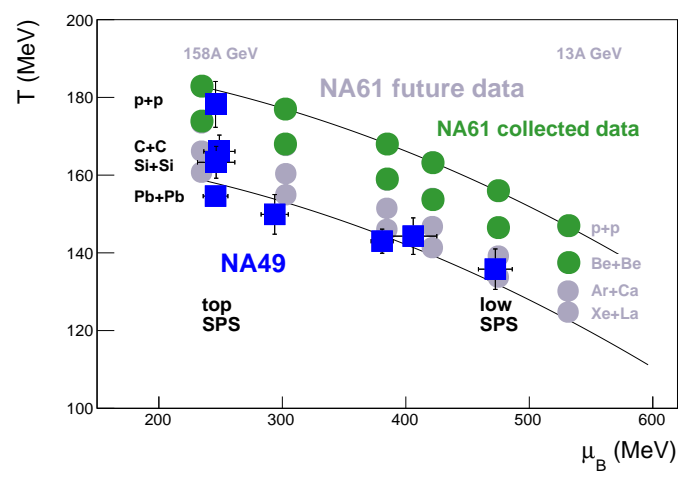

Figure 4: Estimated (NA49) and expected (NA61) chemical freeze-out points to ref. [13] and parameterizations therein. uniform way. The second method $(d E / d x$ analysis) relies the measurements of energy loss $d E / d x$ in the active volume of the TPCs, and in the momentum range of the relativistic rise of $d E / d x$. The results on spectra are corrected for particles from weak decays (feed-down) and detector effects (acceptance, reconstruction inefficiency) using Monte-Carlo simulations. Out of target interactions are subtracted using events recorded with empty liquid hydrogen target.

Examples of 2D (rapidity and transverse momentum) spectra of $\pi^{-}$(from the $h^{-}$method), and $\pi^{+}, K^{-}, p$ (from the $d E / d x$ method) are presented in Fig. 5 for $p+p$ interactions at 158 $\mathrm{GeV} / \mathrm{c}$. Those 2D spectra allow to obtain corresponding 1D spectra of rapidity or $m_{T}$. Examples of rapidity distributions of $\pi^{-}, \pi^{+}, K^{-}$, and $p$ for $p+p$ collisions at $158 \mathrm{GeV} / \mathrm{c}$ in selected $p_{T}$ bins are shown in Fig. 6 and compared to published NA49 data [17]. It can be seen that NA61 results from different methods $\left(d E / d x\right.$ and $\left.h^{-}\right)$and NA49 results agree quite well.

Transverse mass spectra of negatively charged pions in $p+p$ collisions are presented in Fig. 7 (left). The spectra are approximately exponential in $p+p$ collisions, but the right panel shows

\footnotetext{
${ }^{2}$ NA49 data indicate [13] that at the top SPS energy $\mu_{B}$ does not depend on the system size $(C+C, S i+S i, P b+P b)$. Therefore, the $\mu_{B}$ values for $p+p$ are also displayed and assumed to be the same as for $P b+P b$.
} 

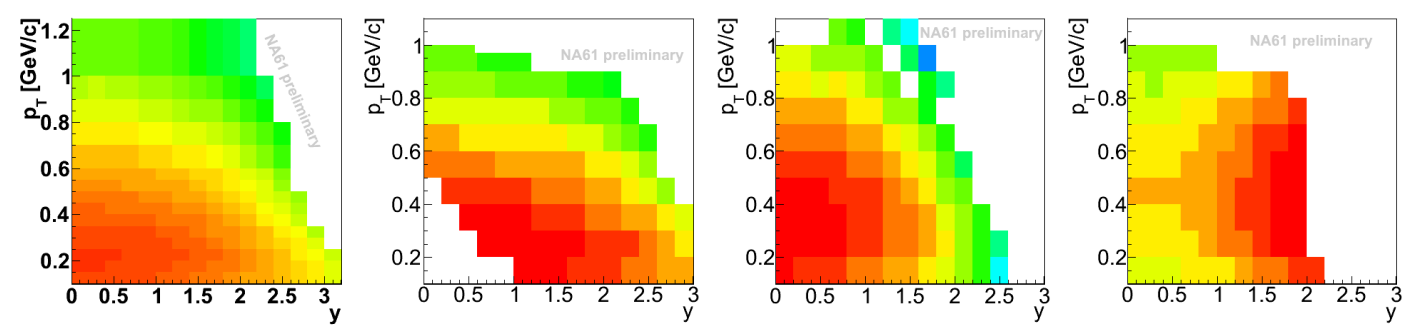

Figure 5: $2 D$ spectra of (from the left): $\pi^{-}$(from $h^{-}$method), and $\pi^{+}, K^{-}, p$ (from $d E / d x$ based identification) in $p+p$ interactions at $158 \mathrm{GeV} / \mathrm{c}$.
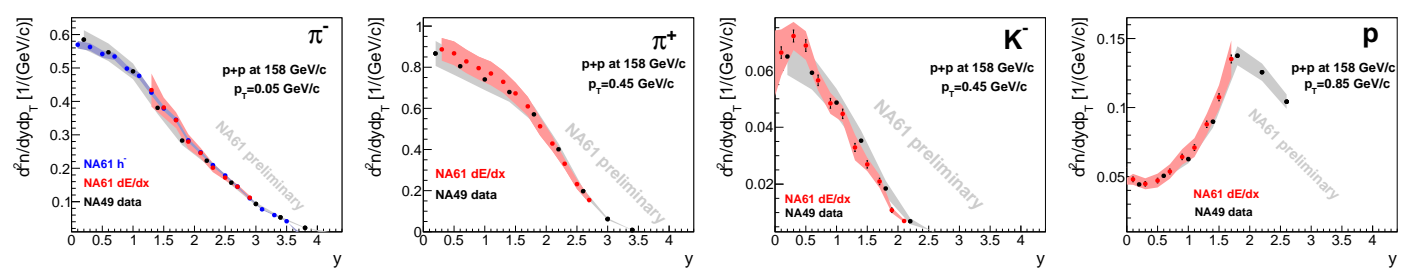

Figure 6: Comparison of NA61 rapidity spectra with NA49 results [17] for inelastic $p+p$ collisions at $158 \mathrm{GeV} / \mathrm{c}$. Shaded bands indicate systematic uncertainties.
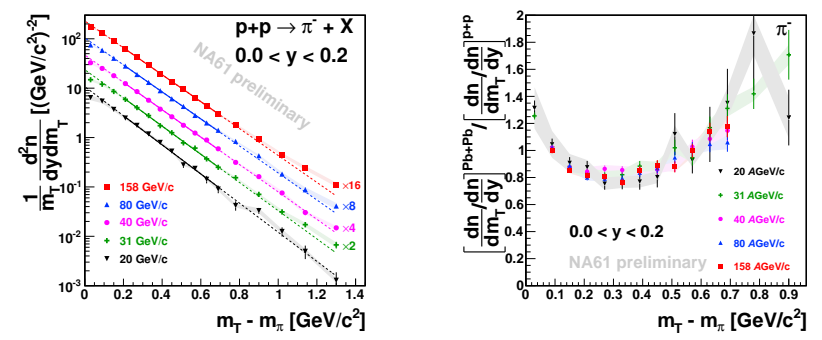

Figure 7: Transverse mass spectra of $\pi^{-}$in $p+p$ collisions (left), and ratio between yields in central $\mathrm{Pb}+\mathrm{Pb}(\mathrm{NA49}[9])$ and $p+p$ (NA61) interactions (right). Results are for mid-rapidity $0.0<y<0.2$.

that the shape differs significantly between $p+p$ and central (7\% and 5\%) $P b+P b$ [9] collisions (mainly due to transverse collective flow in $P b+P b$ ). The ratio between yields normalized to the number of wounded nucleons in $P b+P b$ and $p+p$ collisions shows no significant change with collision energy in the studied $m_{T}$ range. The same conclusions can also be drawn from spectra of protons (Fig. 8). The shape differs significantly between $p+p$ and central $(7 \%, 5 \%)$ $P b+P b[18]$ collisions due to transverse collective flow in $P b+P b$, but in first approximation the differences do not change with collision energy. A similar analysis was also performed for negatively charged kaons. However, the kinematic range of the kaon $d E / d x$ identification does not reach midrapidity for lower energies. It will soon be supplemented by the analysis of ToF signals. Mid-rapidity transverse mass spectra in $p+p$ and in NA49 $P b+P b$ collisions (for both systems the same set of particles was used) were fitted with a simplified Blast-Wave Model [19] assuming a constant transverse velocity profile. The resulting kinetic freeze-out temperatures $(T)$ and transverse velocities $\left(\beta_{T}\right)$ are shown in Fig. 9.

The results shown in Figs 5, 6, 7, and 8 are an important step towards obtaining $p+p$ reference data for the study of the properties of the onset of deconfinement by looking for the kink, horn, step, 

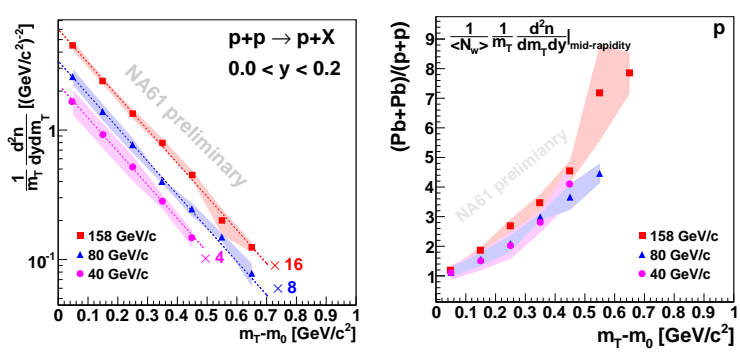

Figure 8: Transverse mass spectra of protons in $p+p$ collisions (left), and ratio between yields in central $P b+P b(N A 49$ [18]) and $p+p$ (NA61) interactions (right). Results are for mid-rapidity. For $\mathrm{Pb}+\mathrm{Pb}$ data y intervals were: $-0.32<y<0.80$ for $40 \mathrm{~A} \mathrm{GeV/c},-0.37<y<0.30$ for $80 \mathrm{~A}$ $\mathrm{GeV} / \mathrm{c},-0.50<y<-0.1$ for $158 \mathrm{~A} \mathrm{GeV/c}$.
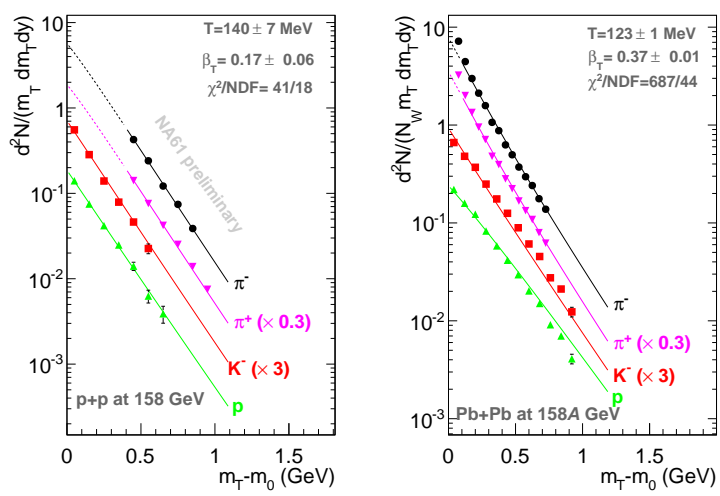

Figure 9: Mid-rapidity transverse mass spectra in $p+p$ and $N A 49 P b+P b$ collisions fitted with a simplified Blast-Wave Model [19].

and dale structures $[14,15]$ in collisions of light and intermediate mass nuclei $(B e+B e, A r+C a$, $X e+L a)$. Figure 10 (left) presents the kink plot of charged pion multiplicity $\left(\langle\pi\rangle=1.5\left(\left\langle\pi^{+}\right\rangle+\right.\right.$ $\left.\left.\left\langle\pi^{-}\right\rangle\right)\right)^{3}$ normalized to the number of wounded nucleons versus Fermi variable $F\left(F \approx\left(s_{N N}\right)^{1 / 4}\right)$. In the Statistical Model of the Early Stage [14] this ratio is proportional to the effective number of degrees of freedom $(N D F)$ to the power of $1 / 4$. For central $A+A$ collisions $(P b+P b$ for SPS or $A u+A u$ for AGS and RHIC) a change of slope around $30 A \mathrm{GeV} / \mathrm{c}$ is visible. Such an increase is not observed for $p+p(\bar{p})$ reactions. The increase in $N D F$, when going from hadron gas to QGP, may be interpreted as a consequence of the activation of partonic degrees of freedom. The ratio $\langle\pi\rangle /\left\langle N_{W}\right\rangle$ increases with energy slower in $p+p$ than in central $P b+P b$ collisions and these two dependencies cross each other at about $40 \mathrm{~A} \mathrm{GeV/c} \mathrm{beam} \mathrm{momentum.} \mathrm{In} \mathrm{Fig.} 10$ (middle-left) the inverse slope parameters $T$ of $m_{T}$ spectra at the SPS energies show different behavior in central $P b+P b$ (step) than in $p+p$ (smooth increase) reactions. According to ref.[21] the sound velocity $c_{s}$ is related within hydrodynamical models to the width of the rapidity distribution $\sigma_{y}$ of pions:

$$
\sigma_{y}^{2}\left(\pi^{-}\right)=\frac{8}{3} \frac{c_{s}^{2}}{1-c_{s}^{4}} \ln \left(\sqrt{s_{N N}} / 2 m_{p}\right)
$$

Figure 10 (right) shows the sound velocities obtained from the widths of $\pi^{-}$rapidity spectra pre-

\footnotetext{
${ }^{3}$ Here we used $\langle\pi\rangle=\frac{3\left\langle\pi^{-}\right\rangle+1}{2}[20]$.
} 

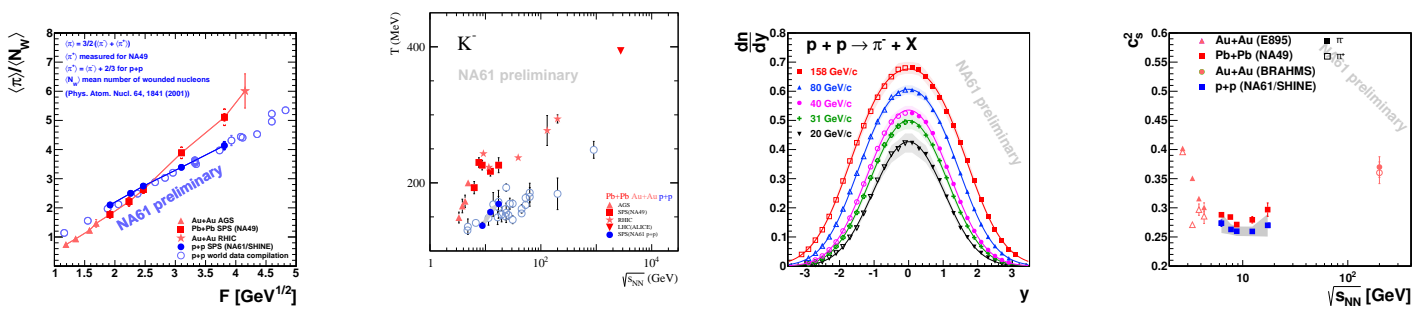

Figure 10: Left: Energy $(F)$ dependence of the mean pion multiplicity per wounded nucleon in full phase space $(4 \pi)$. Middle-left: Energy dependence of inverse slope parameters $T$ of $m_{T}$ spectra. Middle-right: $m_{T}$-integrated $\pi^{-}$rapidity spectra fitted with a sum of two symmetrically displaced Gaussian functions. Right: Energy dependence of sound velocities obtained from widths of $\pi^{-}$rapidity spectra.

sented in Fig. 10 (middle-right). Lattice QCD calculations [22] suggest that the minimum of the sound velocity can be attributed to the phase transition. In Fig. 10 (right) such a minimum (dale) can be seen not only for $A+A$ collisions but also in $p+p$ data.

\subsection{Chemical (particle type) and multiplicity fluctuations}

In the NA61 experiment we used the $\Phi$ measure and the scaled variance $\omega$ of multiplicity fluctuations to study chemical and multiplicity fluctuations, respectively. The scaled variance [23], is defined as:

$$
\omega_{i}=\frac{\left\langle N_{i}^{2}\right\rangle-\left\langle N_{i}\right\rangle^{2}}{\left\langle N_{i}\right\rangle}
$$

where $i$ is the particle type $(\pi, K, p)$, and $\left\langle N_{i}\right\rangle,\left\langle N_{i}^{2}\right\rangle$ are the first and the second moments of the multiplicity distribution of particle type $i$. The scaled variance of multiplicity distribution is an intensive measure of fluctuations (independent of volume), and equals 1 for a Poisson multiplicity distribution. Unfortunately, it depends on volume fluctuations which may distort comparisons of $p+p$ to $P b+P b$ data obtained in wide centrality bins. Chemical (particle type) fluctuations were analyzed using the $\Phi_{i j}$ measure [23] defined as:

$$
\Phi_{i j}=\frac{\sqrt{\left\langle N_{i}\right\rangle\left\langle N_{j}\right\rangle}}{\left\langle N_{i}+N_{j}\right\rangle} \cdot\left[\sqrt{\Sigma^{i j}}-1\right],
$$

where $\Sigma^{i j}=\left[\left\langle N_{i}\right\rangle \cdot \omega_{j}+\left\langle N_{j}\right\rangle \cdot \omega_{i}-2 \cdot\left(\left\langle N_{i} N_{j}\right\rangle-\left\langle N_{i}\right\rangle\left\langle N_{j}\right\rangle\right)\right] /\left\langle N_{i}+N_{j}\right\rangle$ and $i, j$ indicate two different particle types. $\Phi_{i j}$ is a strongly intensive measure of fluctuations (independent of volume and volume fluctuations). For independent particle emission $\Phi_{i j}$ equals 0 .

Experimental results on chemical fluctuations of identified particle multiplicities may be distorted by incomplete particle identification. Therefore, measurements from NA49 and NA61 as presented here were corrected for misidentification using the unfolding procedure of the identity method [24]. Finally, as fluctuations cannot be corrected for the limited acceptance the results are presented both for the NA61 acceptance [25] and a restricted common NA49/NA61 phase space region.

Figure 11 (left) shows the scaled variances of $\pi, K$, and $p+\bar{p}$ (for both charges, i.e. $K=$ $K^{+}+K^{-}, \pi=\pi^{+}+\pi^{-}$) measured by NA61 in $p+p$ collisions. Additionally, $\omega_{N_{c h}}$ was calculated directly by track counting for all charged (only electrons were removed by $d E / d x$ cut). Figure 11 

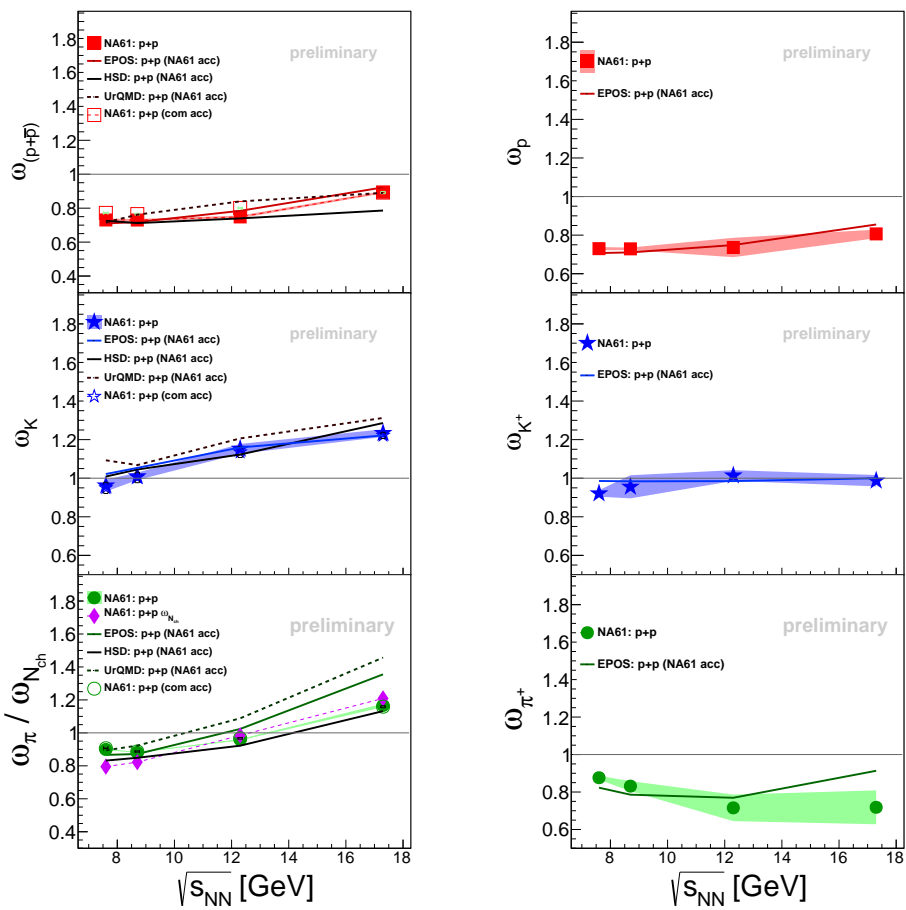

Figure 11: Scaled variance $\omega$ of the multiplicity distributions in inelastic $p+p$ interactions at $31,40,80$, and $158 \mathrm{GeV/c}$. See [25] for definitions of NA61 acceptance and common (NA49 and NA61) phase space region.

(right) shows the scaled variances for positively charged particles. The values of $\omega$ for $p+\bar{p}$ and $p$ are smaller than 1 probably due to baryon number conservation and very close to each other due to the small fraction of antiprotons produced in the interactions. The scaled variance for charged kaons $\left(K^{+}+K^{-}\right)$is higher than 1 probably due to strangeness conservation. This interpretation is supported by the observation of $\omega_{K^{+}}$values which are close to 1 and smaller than $\omega_{K}$ (suggesting that strangeness conservation contributes to $\left.\omega_{K}\right)$. The increase of $\omega_{\pi}$ with energy probably reflects the increase of $\omega_{N_{c h}}$ measured in full phase-space (a linear increase of $\omega_{N_{c h}}$ with $\left\langle N_{c h}\right\rangle$ for $p+p$ collisions in full phase-space was shown in ref. [26]). The values of $\omega_{\pi^{+}}<\omega_{\pi}$ are possibly due to charge conservation. For higher SPS energies $\omega_{\pi}$ and $\omega_{N_{c h}}$ in $p+p$ data are very close to each other. For lower energies they differ due to the significant fraction of protons among all charged particles. The results on multiplicity fluctuations in $p+p$ interactions agree with predictions of the EPOS and HSD models.

Figure 12 shows two particle chemical (between particle types) fluctuations as a function of interaction energy. The left panel shows combinations of both charges, the right panel only of positively charged particles. The values of $\Phi_{\pi(p+\bar{p})}$ and $\Phi_{\pi^{+} p}$ are negative for all studied energies. This is most probably due to charge conservation and resonance decays (see also [27]). A similar but weaker tendency is also observed by NA49 in $P b+P b$ data [28]. In $p+p$ collisions $\Phi_{\pi K}$ is higher than zero probably due to strangeness conservation $\left(\Phi_{\pi^{+} K^{+}}\right.$being close to zero supports this interpretation). For $p+p$ collisions $\Phi_{\pi K}$ slightly increases with energy, but such an effect is not visible for $P b+P b$ interactions of NA49. A very weak increase of $\Phi_{(p+\bar{p}) K}$ with energy 

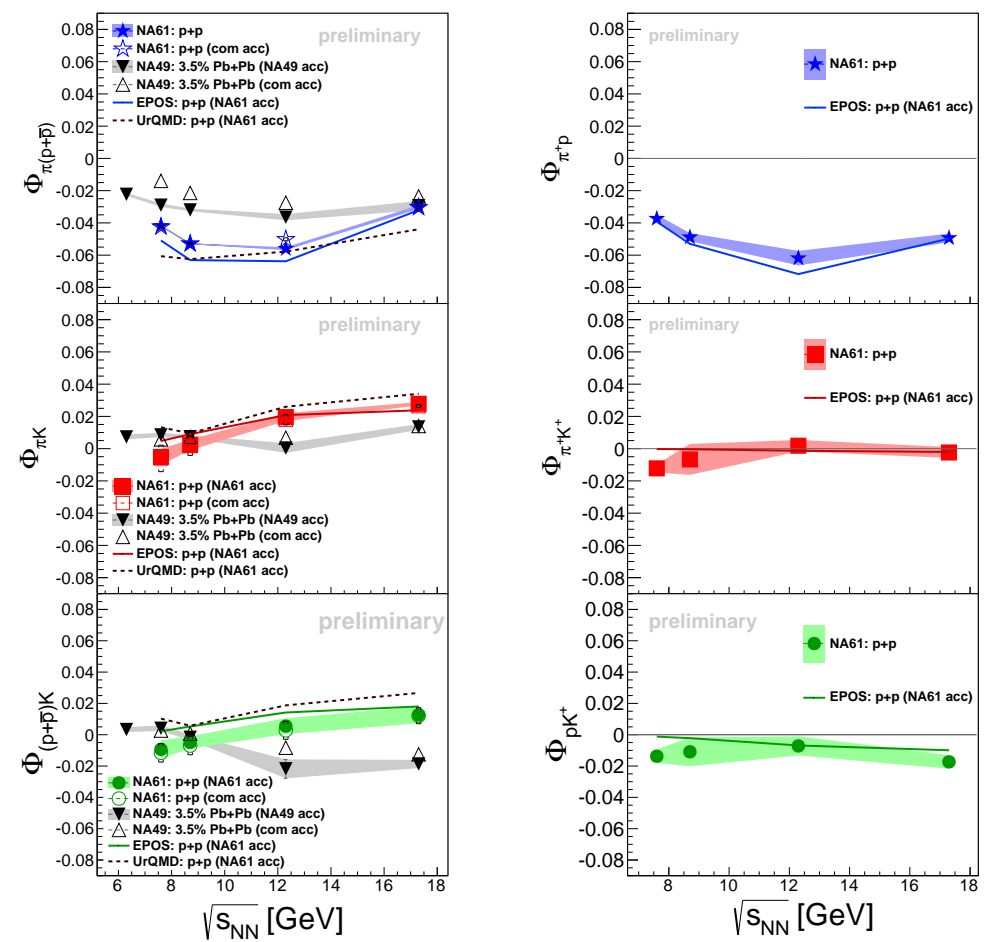

Figure 12: Chemical (particle type) fluctuations in inelastic $p+p$ interactions at 31, 40, 80, and 158 GeV/c. NA61 $p+p$ data are compared to those obtained by NA49 in Pb + Pb collisions [28]. See [25] for definitions of NA61 acceptance, NA49 acceptance, and selected common (NA49 and NA61) phase space region.

can be seen in $p+p$ data, whereas for $P b+P b$ data $\Phi_{(p+\bar{p}) K}$ decreases with energy. For both systems $\Phi_{(p+\bar{p}) K}$ crosses zero at medium SPS energies. Finally, we observe no significant energy dependence of $\Phi_{p K^{+}}$in the NA61 data. The EPOS and UrQMD model predictions reproduce $p+p$ data reasonably well.

\subsection{Transverse momentum fluctuations}

In the NA61 experiment we studied event-by-event transverse momentum fluctuations by use of the strongly intensive measure $\Phi_{p_{T}}$ [29]. In the Wounded Nucleon Model [30] the $\Phi$ measure does not depend on the number of wounded nucleons nor on fluctuations of the number of wounded nucleons. In thermodynamical models $\Phi$ does not depend on volume and volume fluctuations. The $\Phi_{p_{T}}$ measure was already used by NA49 to study system size [31] and energy [32] dependence of transverse momentum fluctuations. The data sets recorded by NA61 in 2009 allow to study $\Phi_{p_{T}}$ in inelastic $p+p$ collisions at 20,31, 40,80, and $158 \mathrm{GeV} / \mathrm{c}$. The $\Phi_{p_{T}}$ measure can be expressed using event quantities $[33,34]$ :

$$
\Phi_{p_{T}} \equiv \sqrt{\frac{\left\langle X^{2}\right\rangle}{\langle N\rangle}-\frac{2\langle X\rangle\langle N X\rangle}{\langle N\rangle^{2}}+\frac{\langle X\rangle^{2}\left\langle N^{2}\right\rangle}{\langle N\rangle^{3}}}-\sqrt{\frac{\left\langle X_{2}\right\rangle}{\langle N\rangle}-\frac{\langle X\rangle^{2}}{\langle N\rangle^{2}}},
$$

where:

$$
X=\sum_{i=1}^{N} p_{T i}, \quad X_{2}=\sum_{i=1}^{N}\left(p_{T_{i}^{2}}^{2}\right),
$$




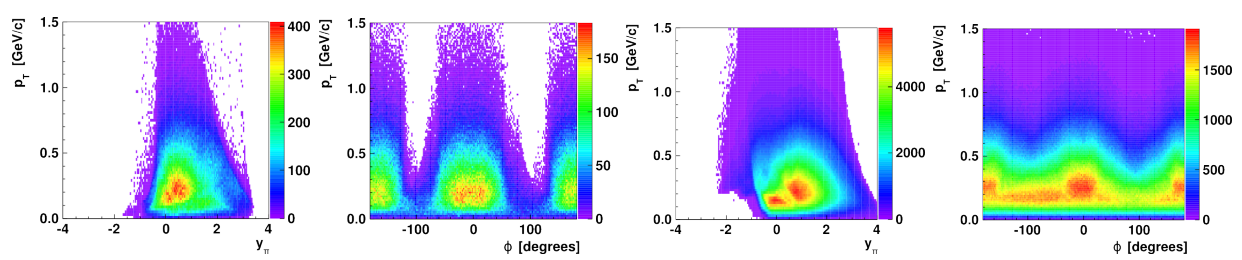

Figure 13: Particle population in the acceptance used by NA61 to study $p_{T}$ fluctuations and two-particle correlations in $(\Delta \eta, \Delta \phi)$ : the two left plots for $20 \mathrm{GeV} / \mathrm{c}$, the two right plots for $158 \mathrm{GeV} / \mathrm{c}$ $p+p$ interactions.
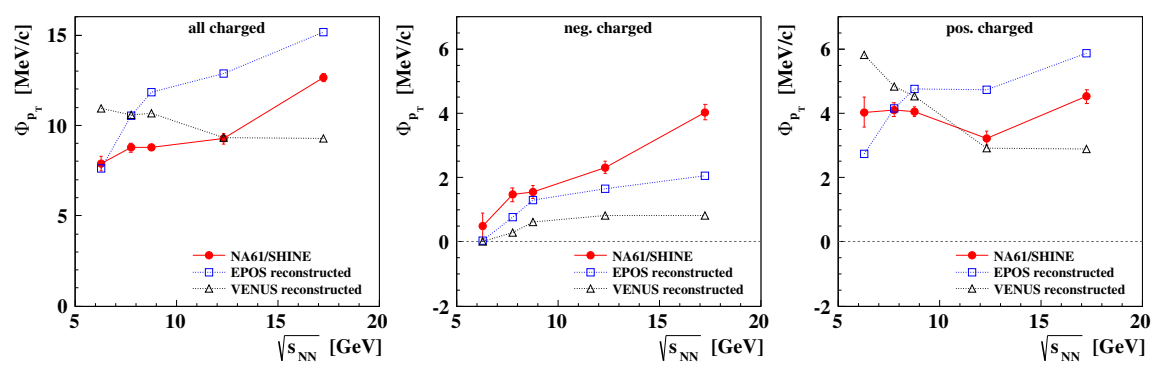

Figure 14: Pilot results on $\Phi_{p_{T}}$ versus energy for $p+p$ interactions corrected for non-target interactions. NA61 data are compared to predictions of VENUS and EPOS models, which were processed through full detector simulation. Only statistical errors are shown.

and $N$ is the multiplicity of accepted particles in the event.

The acceptance used in NA61 for $p_{T}$ fluctuations is presented in Fig. 13. Figure 14 shows pilot results on $\Phi_{p_{T}}$ calculated for all charged, negatively charged, and positively charged particles produced in $p+p$ collisions at 20-158 GeV/c beam momentum. The results are corrected for the effects of non-target interactions (for the procedure see ref. [35]; however, a more advanced and comprehensive correction method is currently being developed [36]). The non-target interaction correction was done by using data sets recorded with empty liquid hydrogen target. The results corrected not only for the contamination of non-target interactions but also for detector inefficiencies, contamination of non-vertex tracks (trigger bias), etc. will be coming soon.

Figure 14 shows that for all charge combinations transverse momentum fluctuations are higher then zero. In order to compare NA61 pilot results with NA49 data, more restrictive NA49 cuts were applied to NA61 data. Due to the high density of tracks at mid-rapidity in $P b+P b$ collisions the analysis of NA49 was limited to the forward-rapidity region $\left(1.1<y_{\pi}<2.6\right.$, where $y_{\pi}$ is the rapidity calculated in the center-of-mass reference system, assuming the pion mass). Moreover, a common azimuthal region was used for all energies (see [32] for details) meaning that the azimuthal acceptance of the detector was strongly reduced at higher energies. Figure 15 shows that by applying NA49 cuts $\Phi_{p_{T}}$ in $p+p$ interactions decreases (mainly because of the narrower rapidity range). The NA61 experiment plans to extend its physics program to repeat and complement the NA49 measurements in $P b+P b$ collisions (see Fig.1). The new He beam pipes reduce the number of $\delta$-electrons in the VTPCs by a factor of 10 allowing to extend the analysis towards mid-rapidity. The right panel of Fig. 15 presents a comparison of $\Phi_{p_{T}}$ for $p+p$ (NA61) and $P b+P b$ (NA49) collisions within the NA49 phase-space selection cuts. No significant difference, as well as, no indications of the critical point (hill of fluctuations) is observed in the energy scan of $\mathrm{Pb}+\mathrm{Pb}$ and 

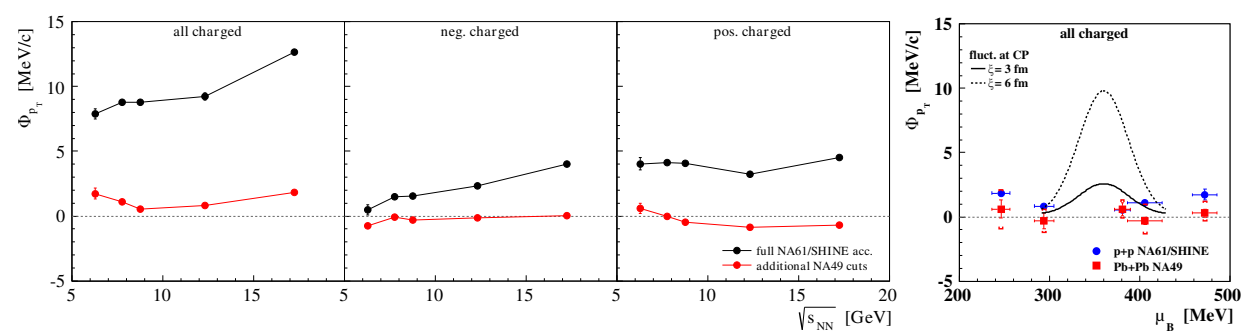

Figure 15: Three first plots: pilot results on $\Phi_{p_{T}}$ versus energy for $p+p$ interactions using the full NA61 acceptance (see Fig.13) and with additional NA49 phase space cuts (see [32]). Rightmost plot: $\Phi_{p_{T}}$ versus $\mu_{B}$ for $p+p$ (NA61) and $P b+P b$ (NA49 [32]) collisions in the phase space region selected by $N A 49$ (see [37] for details of CP predictions).

$p+p$ interactions. We are waiting for the results from $B e+B e, A r+C a$, and $\mathrm{Xe}+\mathrm{La}$ interactions.

\subsection{Two-particle correlations in $(\Delta \eta, \Delta \phi)$}

Two-particle correlations in pseudo-rapidity $\eta$ and azimuthal angle $\phi$ were studied extensively both at RHIC and LHC. Such measurements help to disentangle different sources of correlations: jets, flow, resonance decays, quantum statistics effects, conservation laws, etc. We performed a two-particle correlation analysis for "full target" $p+p$ interactions (without subtraction of the small contamination by non-target interactions) at $158 \mathrm{GeV} / \mathrm{c}$ beam momentum. Correlations are calculated as a function of the difference in pseudo-rapidity and azimuthal angle between two particles in the same event:

$$
\Delta \eta=\left|\eta_{1}-\eta_{2}\right| \quad \Delta \phi=\left|\phi_{1}-\phi_{2}\right|
$$

The correlation function ${ }^{4}$ is defined as:

$$
\begin{gathered}
C(\Delta \eta, \Delta \phi)=\frac{N_{\text {mixed }}^{\text {pairs }}}{N_{\text {data }}^{\text {pairs }}} \frac{S(\Delta \eta, \Delta \phi)}{M(\Delta \eta, \Delta \phi)}, \\
S(\Delta \eta, \Delta \phi)=\frac{d^{2} N^{\text {signal }}}{d \Delta \eta d \Delta \phi} ; \quad M(\Delta \eta, \Delta \phi)=\frac{d^{2} N^{\text {mixed }}}{d \Delta \eta d \Delta \phi}
\end{gathered}
$$

In order to reduce statistical errors the correlation function was folded around $\Delta \phi=0$ : when $\Delta \phi>\pi$ then $\Delta \phi=2 \pi-\Delta \phi$. To allow comparisons with RHIC and LHC results pseudo-rapidity was calculated in the center-of-mass (CMS) reference system. The transformation from the NA61 laboratory system to the CMS assumed pion mass for all produced particles.

Examples of correlation functions in $p+p$ interactions at $158 \mathrm{GeV} / \mathrm{c}$ are shown in Fig. 16. In addition to the "inclusive" results, shown in Fig. 16, correlation functions were also computed for narrow multiplicity bins. By dividing the analysis into multiplicity bins one can better study contributions of certain phenomena to the inclusive correlations (e.g. resonance decays) - see also [38]. The correlation function was calculated for multiplicities of 2, 3, 4, and 5, and separately for all charged, positively charged, and negatively charged particles (Fig. 17). A maximum for all

\footnotetext{
${ }^{4}$ The correlation function ratio was calculated and normalized in the $\Delta \eta$ region: $0<\Delta \eta<3$. Event and track cuts were chosen to select only inelastic interactions with particles produced in strong and EM processes within the NA61 acceptance.
} 

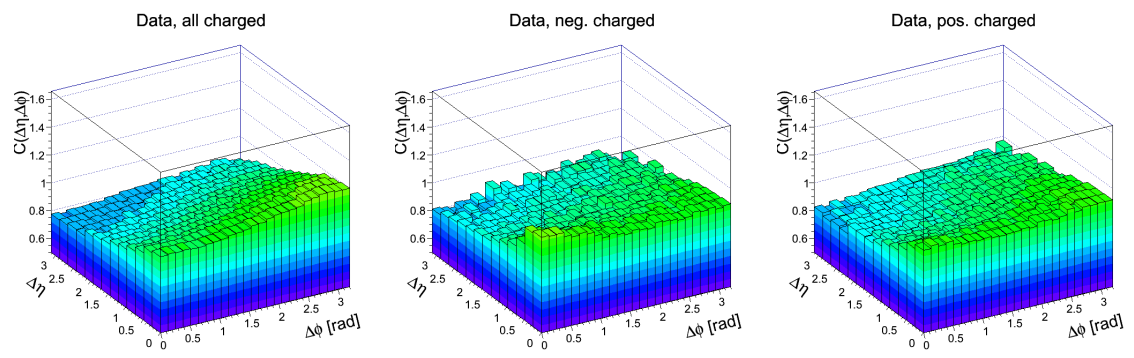

Figure 16: Correlation function $C(\Delta \eta, \Delta \phi)$ in $p+p$ interactions at $158 \mathrm{GeV} / \mathrm{c}$.
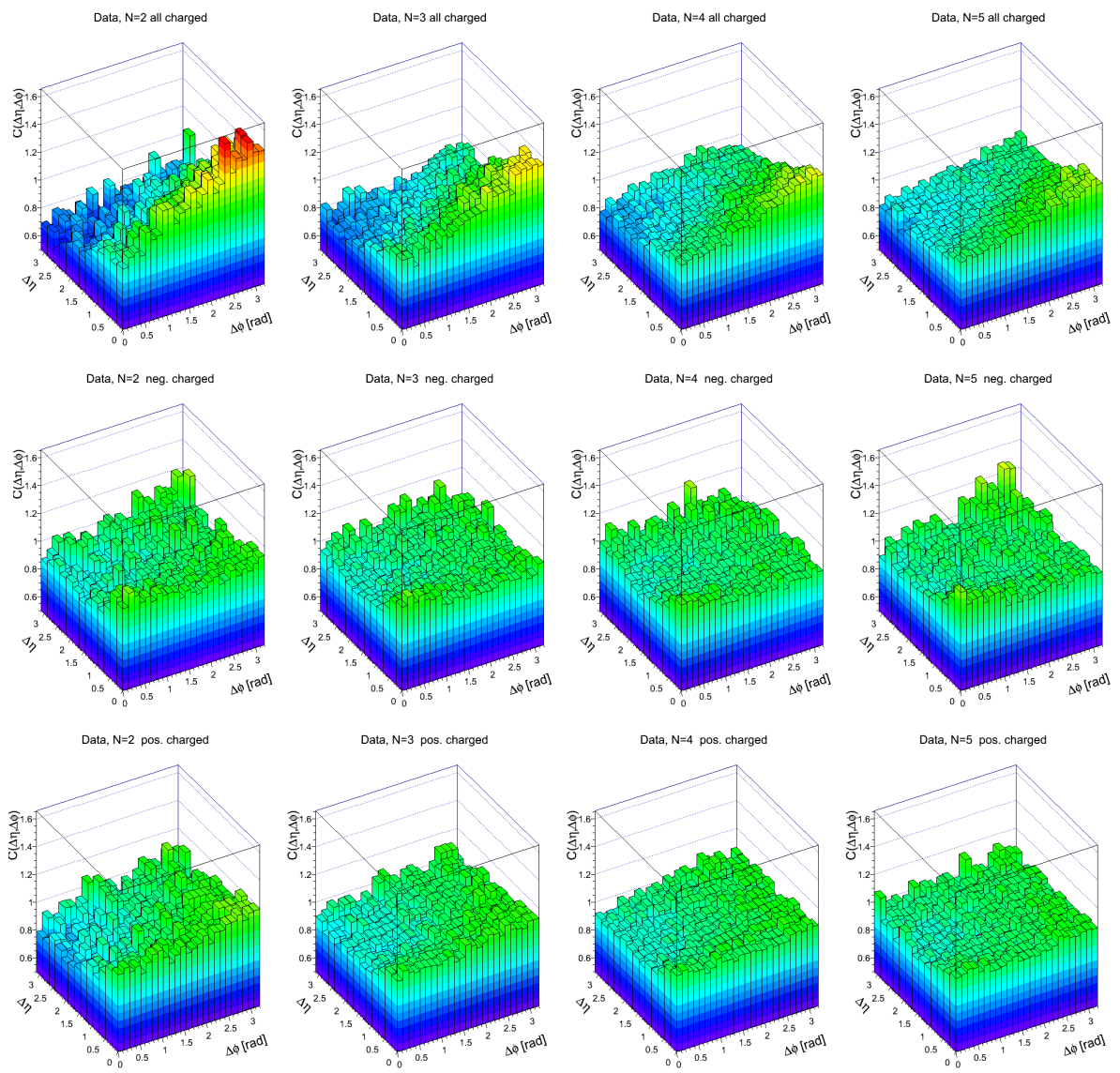

Figure 17: Correlation function $C(\Delta \eta, \Delta \phi)$ in $p+p$ collisions at $158 \mathrm{GeV} / \mathrm{c}$ for multiplicities of all charged, positively charged or negatively charged particles equal to 2, 3, 4, and 5 .

charged and positively charged particles at $(\Delta \eta, \Delta \phi)=(0, \pi)$ can be seen in Fig. 16. It is probably caused by resonance decays (mostly $\Delta^{++}$for positively charged) and momentum conservation. This maximum is better visible for multiplicity selections (Fig. 17). As there are practically no resonances which decay into two negatively charged particles, the very low peak for negatively charged particles at $(0, \pi)$ is probably connected with momentum conservation. For all three charge combinations one observes an enhancement around $(0,0)$ which is probably due to effects of quantum statistics and Coulomb interactions. For negatively charged particles we also see a hill at $(3, \pi)$ for multiplicity selected correlations. This is probably due to global momentum conservation. Results from the VENUS and EPOS models (not shown in this paper) exhibit similar structures except the 
hill around $(0,0)$. This is not surprising since VENUS and EPOS do not simulate Coulomb and quantum effects. A similar analysis is currently being performed on NA61 $p+p$ data at all five collision energies and on $P b+P b$ data of NA49.

\section{Summary}

NA61/SHINE at the CERN SPS pursues different programs: particle production measurements for neutrino and cosmic-ray experiments, studies of ion collisions and investigations of nuclear effects in low and high transverse momentum particle production.

Pion spectra from $p+C$ interactions at $31 \mathrm{GeV} / \mathrm{c}$ were published and are already used to improve beam neutrino flux predictions in the T2K experiment and to adjust models used in $v$ beam property predictions and simulations of cosmic-ray induced air showers. Positively charged kaon spectra in $p+C$ at $31 \mathrm{GeV} / \mathrm{c}$ were also published; numerous preliminary results $\left(K_{S}^{0}, \Lambda\right.$, proton yields in $p+C$ collisions at $31 \mathrm{GeV} / \mathrm{c}, h^{+}, h^{-}$yields in $\pi^{-}+\mathrm{C}$ interactions at $158 \mathrm{GeV} / \mathrm{c}$ and 350 $\mathrm{GeV} / \mathrm{c}$ ) are released.

First results from the energy scan of $p+p$ collisions at $20-158 \mathrm{GeV} / \mathrm{c}$ were presented. $\pi^{-}$ spectra, as well as $\pi^{+}, K^{-}$and $p$ spectra obtained at $40-158 \mathrm{GeV} / \mathrm{c}$, were shown. Agreement was found with published results at $158 \mathrm{GeV} / \mathrm{c}$. The new measurements provide an improved reference for the studies of the properties of the onset of deconfinement. Interestingly, the dale structure seems to be also present in $p+p$ interactions.

Transverse momentum, multiplicity and chemical (particle composition) fluctuations were studied in $p+p$ collisions at 20(31)-158 GeV/c. Conservation laws play an important role in chemical and multiplicity fluctuations in $p+p$ collisions. Fluctuations of $p+\bar{p}$ with respect to $K$ show different behavior in $p+p$ (NA61) and in $P b+P b$ (NA49) collisions, but in both systems they change sign at medium SPS energies. Models (EPOS, HSD, UrQMD) reproduce the tendencies observed in NA61 $p+p$ data. Transverse momentum fluctuations (pilot results) in $p+p$ interactions at 20-158 GeV/c are significant in the acceptance of NA61. They are consistent with zero when additional cuts (forward rapidity) are applied, as had to be used in the energy scan of $P b+P b$ collisions in NA49. Improvements of the NA61 detector (i.e. He beam pipes) will allow to study fluctuations in the complete rapidity acceptance of NA61 also for heavy nuclei.

ACKNOWLEDGMENTS: This work was partially supported by the the National Science Centre, Poland grant DEC-2011/03/B/ST2/02617 and grant 2012/04/M/ST2/00816.

\section{References}

[1] https://na61.web.cern.ch/na61/xc/index.html

[2] S.V. Afanasiev et al. [NA49 Collaboration], Nucl. Instrum. Meth. A 430, 210 (1999).

[3] K. Abe et al. [T2K Collaboration], Phys. Rev. Lett. 107, 041801 (2011).

[4] N. Abgrall et al. [NA61/SHINE Collaboration], Phys. Rev. C 84, 034604 (2011).

[5] V. Uzhinsky, arXiv:1107.0374

[6] V. Uzhinsky, arXiv:1109.6768 
[7] N. Abgrall et al. [NA61/SHINE Collaboration], Phys. Rev. C 85, 035210 (2012).

[8] N. Abgrall et al. [NA61/SHINE Collaboration], Nucl. Instrum. Meth. A 701, 99 (2013).

[9] S.V. Afanasiev et al. [NA49 Collaboration], Phys. Rev. C 66, 054902 (2002);

C. Alt et al. [NA49 Collaboration], Phys. Rev. C 77, 024903 (2008).

[10] Z. Fodor, S.D. Katz, JHEP 0404, 050 (2004).

[11] A. Li et al. RIKEN-BNL Workshop On Fluctuations, Correlations and RHIC Low Energy Runs, Oct. 3-5 (2011), http://www.bnl.gov/fcrworkshop/

[12] S. Datta, R.V. Gavai, S. Gupta, arXiv: 1210.6784

[13] F. Beccatini, J. Manninen, M. Gazdzicki, Phys. Rev. C 73, 044905 (2006).

[14] M. Gazdzicki, M. Gorenstein, Acta Phys. Polon. B 30, 2705 (1999).

[15] M. Gazdzicki, M. Gorenstein, P. Seyboth, Acta Phys. Polon. B 42307 (2011).

[16] M. Stephanov, K. Rajagopal, E.V. Shuryak, Phys. Rev. D 60, 114028 (1999).

[17] C. Alt et al. [NA49 Collaboration], Eur. Phys. J. C 45, 343 (2006);

T. Anticic et al. [NA49 Collaboration], Eur. Phys. J. C 65, 9 (2010);

T. Anticic et al. [NA49 Collaboration], Eur. Phys. J. C 68, 1 (2010).

[18] C. Alt et al. [NA49 Collaboration], Phys. Rev. C 73, 044910 (2006).

[19] E. Schnedermann, J. Sollfrank, U. Heinz, Phys. Rev. C 48, 2462 (1993).

[20] A.I. Golokhvastov, Phys. Atom. Nucl. 64, 1841 (2001).

[21] V. Shuryak, Yad. Fiz. 16, 395 (1972).

[22] S. Borsanyi et al., JHEP 1011, 077 (2010).

[23] M.I. Gorenstein, M. Gazdzicki, Phys. Rev. C 84, 014904 (2011).

[24] M. Gazdzicki, K. Grebieszkow, M. Mackowiak, S. Mrowczynski, Phys. Rev. C 83, 054907 (2011); M.I. Gorenstein, Phys. Rev. C 84, 024902 (2011);

A. Rustamov, M.I. Gorenstein, Phys. Rev. C 86, 044906 (2012).

[25] https://edms.cern.ch/document/1237791/1

[26] H. Heiselberg, Phys. Rept. 351, 161 (2001).

[27] C. Alt et al. [NA49 collaboration], Phys. Rev. C 70, 064903 (2004).

[28] A. Rustamov, these proceedings.

[29] M. Gazdzicki, S. Mrowczynski, Z. Phys. C 54, 127 (1992).

[30] A. Bialas, M. Bleszynski, W. Czyz, Nucl. Phys. B 111, 461 (1976).

[31] T. Anticic et al. [NA49 Collaboration], Phys. Rev. C 70, 034902 (2004).

[32] T. Anticic et al. [NA49 Collaboration], Phys. Rev. C 79, 044904 (2009).

[33] S. Mrowczynski, Phys. Lett. B 465, 8 (1999).

[34] F. Liu, A. Tai, M. Gazdzicki, R. Stock, Eur. Phys. J. C 8, 649 (1999).

[35] T. Cetner, K. Grebieszkow [for NA61 Collaboration], Phys. Atom. Nucl. 75, 567 (2012).

[36] T. Czopowicz, these proceedings.

[37] K. Grebieszkow [for NA49 Collaboration], Nucl. Phys. A 830, 547c (2009), and references therein.

[38] A.I. Golokhvastov, Z. Phys. C 64, 301 (1994). 